\title{
Grüneisen parameters: Origin, identity, and quantum refrigeration
}

\author{
Yi-Cong Yu, ${ }^{1}$ Shizhong Zhang, ${ }^{2, *}$ and Xi-Wen Guan $\oplus^{1,3, \dagger}$ \\ ${ }^{1}$ State Key Laboratory of Magnetic Resonance and Atomic and Molecular Physics, Wuhan Institute of Physics and Mathematics, Innovation \\ Academy for Precision Measurement Science and Technology, Chinese Academy of Sciences, Wuhan 430071, China \\ ${ }^{2}$ Department of Physics and HKU-UCAS Joint Institute for Theoretical and Computational Physics at Hong Kong, \\ The University of Hong Kong, Hong Kong, China \\ ${ }^{3}$ Department of Theoretical Physics, Research School of Physics and Engineering, Australian National University, \\ Canberra, ACT 0200, Australia
}

(Received 13 September 2019; revised 16 July 2020; accepted 18 August 2020; published 13 October 2020)

\begin{abstract}
In solid-state physics, the Grüneisen parameter (GP) was first introduced to study the effect of volume change of a crystal lattice on its vibrational frequencies and has since been widely used to investigate the characteristic energy scales of systems associated with the changes of external potentials. However, the GP is less investigated in gas systems and especially strongly interacting quantum gases. Here we report on some general results on the origin of the GP, an identity, and caloric effects in ultracold quantum gases. We prove that there exists a simple identity among three different types of GPs, quantifying the caloric effect induced by variations of volume, magnetic field, and interaction, respectively. Using exact Bethe ansatz solutions, we present a rigorous study of these different GPs and the quantum refrigeration in one-dimensional Bose and Fermi gases. Based on the exact equations of states of these systems, we further obtain analytic results for singular behavior of the GPs and the caloric effects at quantum criticality. We also predict the existence of the lowest temperature for cooling near a quantum phase transition. It turns out that the interaction ramp up and down in quantum gases provide a promising protocol of quantum refrigeration in addition to the usual adiabatic demagnetization cooling in solid-state materials.
\end{abstract}

DOI: 10.1103/PhysRevResearch.2.043066

\section{INTRODUCTION}

The structure of the energy spectrum of a quantum manybody system and its evolution under external perturbation can be used to characterize its possible phases. As an example, the Grüneisen parameter (GP) [1,2], which was introduced by Grüneisen at the beginning of the 20th century in the study of the effect of volume change of a crystal lattice on its vibrational frequencies, has been extensively studied for the exploration of the caloric effect of solids and phase transitions associated with volume change. Similarly, the magnetic GP quantifies the magnetocaloric effect (MCE), establishing the connection between refrigeration and variation of the magnetic field.

So far, the GP has found diverse applications in geophysics [3,4], chemical physics [5,6], high-pressure physics, and plasma physics [7-9]. Recently, experiments have also started to investigate the GP in heavy-fermion systems [10-12], in which the physical properties at low temperatures are dominated by $f$ electrons and their antiferromagnetic

\footnotetext{
*shizhong@hku.hk

†xwe105@wipm.ac.cn

Published by the American Physical Society under the terms of the Creative Commons Attribution 4.0 International license. Further distribution of this work must maintain attribution to the author(s) and the published article's title, journal citation, and DOI.
}

exchange interaction with the conduction electrons [13]. Heavy-fermion metals are extremely sensitive to a small change in pressure, and this pressure sensitivity is reflected in highly enhanced values of the GP [14]. At low temperatures, divergence of the GP stronger than logarithmic upon cooling in the quantum regime is used for experimental identification of quantum critical points $[10,15-18]$.

In fact, there are many formulations of the GP to quantify the degree of anharmonicity of the energy spectrum in response to volume change. The original definition of the GP was introduced by Grüneisen for the Einstein model [1,2],

$$
\Gamma=:-\frac{V}{\omega_{0}} \frac{\partial \omega_{0}}{\partial V}=\frac{V}{C_{V}} \frac{\partial S}{\partial V},
$$

where the excitations in a solid are described by $N$ phonons with the same frequency $\omega_{0} . S$ is the entropy, and $V$ denotes the volume. In quantum statistical physics, the differential forms of the internal energy $E$ and the pressure $p$ can be represented by the fluctuations and covariances of thermodynamic quantities. If we regard the population $a_{i}$ of the $i$ th energy level as a distribution function of a random variable and observable thermal quantities as the expectation value with respect to this distribution, then one can obtain the following differential relations (see the Supplemental Material [19]):

$$
\begin{gathered}
d E=[\operatorname{Cov}(E, E)] d \beta+[-p-\beta \operatorname{Cov}(p, E)] d V, \\
d p=[\operatorname{Cov}(p, E)] d \beta+\left[E^{\prime \prime}+\beta \operatorname{Cov}(p, p)\right] d V,
\end{gathered}
$$


where Cov denotes the covariance. $E^{\prime \prime}=: \sum_{i} a_{i} \partial^{2} \epsilon_{i} / \partial V^{2}$, and $\beta=1 /\left(k_{B} T\right) . k_{B}$ is the Boltzmann constant, and $T$ is the temperature. Then the GP is simply given by

$$
\Gamma=\frac{V \operatorname{Cov}(p, E)}{\operatorname{Cov}(E, E)}=\frac{V d p /\left.d \beta\right|_{V}}{d E /\left.d \beta\right|_{V}} .
$$

Thus, in this case, $\Gamma$ represents the relative importance of energy-pressure covariance and the energy fluctuation in the system. In contrast to the susceptibility (or compressibility) Wilson ratio proposed in [20-22], i.e., the ratio between the magnetization $M$ (or particle number) fluctuation and the energy fluctuation, $R_{W}^{\chi} \propto \frac{\operatorname{Cov}(M, M)}{\operatorname{Cov}(E, E)}\left[\operatorname{or} R_{W}^{\kappa} \propto \frac{\operatorname{Cov}(N, N)}{\operatorname{Cov}(E, E)}\right]$, the cross correlation in Eq. (2) provides additional insights into the system.

There are distinct advantages in studying the GP in dilute quantum gases. Because of the much smaller compressibility, it is much easier to change to the volume of quantum gases by modifying the external potential, and the effects of external magnetic field can be studied via population imbalance. In addition to this, it is also possible to change the interaction directly by using Feshbach resonance $[23,24]$. This possibility suggests a new avenue for studying a novel interacting GP in addition to those defined by changes in volume or magnetic field $[8,11-16,18,25-30]$. Furthermore, we establish an exact identity between these various GPs, making use of the scaling properties of the quantum gas system.

\section{THEORY: GENERALIZATION OF GRÜNEISEN PARAMETERS AND A NEW IDENTITY}

\section{A. Grüneisen parameters in the grand-canonical ensemble}

In theoretical study, it is far more convenient to work in the grand-canonical ensemble, and it is useful to derive the form of $\Gamma$ in the grand-canonical ensemble. Letting $\mu$ be the chemical potential of the system, one finds

$$
\Gamma=V \frac{\left.\frac{d p}{d T}\right|_{V, N}}{\left.\frac{d E}{d T}\right|_{V, N}}=\frac{1}{T} \frac{\frac{\partial^{2} p}{\partial \mu^{2}} \frac{\partial p}{\partial T}-\frac{\partial^{2} p}{\partial \mu \partial T} \frac{\partial p}{\partial \mu}}{\frac{\partial^{2} p}{\partial \mu^{2}} \frac{\partial^{2} p}{\partial T^{2}}-\left(\frac{\partial^{2} p}{\partial \mu \partial T}\right)^{2}} .
$$

In deriving the above equations, we used Maxwell's relations and the homogeneous assumption, i.e., $\Omega=-p V$ [31]. Here the grand-thermal potential is a linear function of the volume by neglecting the surface effect in the thermodynamic limit [32].

There is a widely used effective GP in experiment, defined as the ratio of the thermal expansion parameter $\beta_{T}=\left.\frac{1}{V} \frac{\partial V}{\partial T}\right|_{p, N}$ to the specific heat at a constant volume $[11,12,14,25,30,33]$ :

$$
\Gamma_{\text {eff }}=\frac{\beta_{T}}{c_{V} / V}=\Gamma \frac{\partial^{2} p}{\partial \mu^{2}}\left(\frac{\partial p}{\partial \mu}\right)^{-2}=\Gamma \frac{\kappa}{n^{2}},
$$

where $\kappa$ is the compressibility and $n$ is the density. We denote it as the "eff-GP" since it is not equivalent to the original definition (3). In the above equation, the thermal expansion parameter in the grand-canonical ensemble is given by

$$
\beta_{T}=\left(\frac{\partial^{2} p}{\partial \mu^{2}} \frac{\partial p}{\partial T}-\frac{\partial^{2} p}{\partial \mu \partial T} \frac{\partial p}{\partial \mu}\right)\left(\frac{\partial p}{\partial \mu}\right)^{-2} .
$$

Note that the usefulness of the eff-GP is well established in experiment; see the discussion on its divergent behavior at quantum critical points $[12,14,29]$. However, it is clear that the eff-GP is not a dimensionless parameter and shows different scaling forms at the quantum critical points. To clearly show the dimensionless nature of the Grüneisen parameter, we present another form of the GP [19]:

$$
\Gamma=\frac{\left.V \frac{\partial S}{\partial V}\right|_{N, T}}{\left.T \frac{\partial S}{\partial T}\right|_{N, V}},
$$

which is equivalent to the definition (1)-(3) and is intimately related to the expansionary caloric effect

$$
\left.\frac{\partial T}{\partial V}\right|_{S, N, H}=\frac{T}{V} \Gamma .
$$

There are other parameters, in addition to volume, that can be used to change the state of the system. As an example, the well-known magnetic GP discussed in experiments $[14-16,18]$ can be introduced analogously by replacing the volume $V$ by the magnetic field $H$ in the definition (6),

$$
\Gamma_{\text {mag }}=-\frac{\left.H \frac{\partial S}{\partial H}\right|_{N, T, V}}{\left.T \frac{\partial S}{\partial T}\right|_{N, H, V}} .
$$

Here we added a minus sign following previous work [18,34] and put the magnetic field $H$ in the numerator in order to make the magnetic GP dimensionless. It is straightforward to obtain the explicit form of the magnetic GP in the grand-canonical ensemble,

$$
\Gamma_{\text {mag }}=-\frac{H}{T} \frac{\frac{\partial^{2} p}{\partial \mu^{2}} \frac{\partial^{2} p}{\partial H \partial T}-\frac{\partial^{2} p}{\partial \mu \partial H} \frac{\partial^{2} p}{\partial \mu \partial T}}{\frac{\partial^{2} p}{\partial \mu^{2}}} .
$$

The magnetic GP (8) plays an important role in studies of solid-state materials $[13,18,28,33,35]$. One of the most important features of the magnetic materials is the magnetocaloric effect, related to the magnetocaloric refrigeration (adiabatic demagnetization cooling); see recent developments [16,17]. By the definition of $\Gamma_{\text {mag }}$ in Eq. (8), we further obtain

$$
\left.\frac{\partial T}{\partial H}\right|_{S, N, V}=\frac{T}{H} \Gamma_{\mathrm{mag}},
$$

which establishes an important relation between the magnetocaloric effect and the magnetic GP. Experimentally, it is easier to measure the magnetocaloric effect and, from Eq. (10), obtain $\Gamma_{\text {mag }}$ instead of using its original definition (8). We can obtain the magnetic entropy change $\partial S /\left.\partial H\right|_{N, T, V}$ once we know the value of the specific heat. The magnetic GP contains information free of any material-specific parameter [18].

\section{B. The interacting Grüneisen parameter}

In addition to the usual conjugate variables that one usually encounters in thermodynamics, in ultracold atomic gases, it is also possible to define another set of conjugate variables related to the interaction between atoms. In the case of $s$-wave interacting quantum gases, the low-energy scattering properties are determined entirely by the $s$-wave scattering length $a_{s}$. 
In a one-dimensional (1D) system, the 1D coupling constant $c$ is related to the scattering length $\left(c \propto a_{s}^{-1}\right)$. In reality, it is possible to change the scattering length $a_{s}$ by the Feshbach resonance, and one can define analogously another GP related to interaction,

$$
\Gamma_{\text {int }}=-\frac{\left.c \frac{\partial S}{\partial c}\right|_{N, H, T, V}}{\left.T \frac{\partial S}{\partial T}\right|_{N, H, c, V}}=-\frac{\frac{\partial^{2} p}{\partial \mu^{2}} \frac{\partial^{2} p}{\partial c \partial T}-\frac{\partial^{2} p}{\partial \mu \partial c} \frac{\partial^{2} p}{\partial \mu \partial T}}{\frac{\partial^{2} p}{\partial \mu^{2}} \frac{\partial^{2} p}{\partial T^{2}}-\left(\frac{\partial^{2} p}{\partial \mu \partial T}\right)^{2}} \frac{c}{T} .
$$

The physical significance of $\Gamma_{\text {int }}$ is that it describes the caloric effect due to modification of interaction strength. In particular, in an isentropic process, one can relate the change in temperature to interaction strength given by

$$
\left.\frac{\partial T}{\partial c}\right|_{S, N, V, H}=\frac{T}{c} \Gamma_{\text {int }} .
$$

This is an interaction analog of the magnetocaloric effect. We observe from Eq. (12) that a heat engine and quantum refrigeration can be constructed by tuning the interaction strength in quantum gases. Therefore, the interaction gradient is also capable of cooling the system just like the magnetization gradient cooling [36,37].

\section{An exact identity}

So far we have presented three different GPs, i.e., $\Gamma, \Gamma_{\text {mag }}$, and $\Gamma_{\text {int }}$, which quantify the degrees of anharmonicity of spectral structures in regard to the variations of volume, magnetic field, and interaction strength, respectively. Using the general thermal potential [38], one can find an identity for the three GPs for a dilute system described by the $s$-wave scattering length $a_{s}$ [23]. For these systems, one has the following scaling transformations: $L \rightarrow e^{\lambda} L, c \rightarrow e^{\chi \lambda} c$, where $e^{\lambda}$ is the scaling amplitude and $\chi$ describes the dependences of the coupling constant $c$ on the scattering length, $c \propto a_{s}^{\chi}$; then the Hamiltonian transforms as $H \rightarrow e^{-2 \lambda} H$. As a result, the spectrum transforms as $\epsilon_{n} \rightarrow e^{-2 \lambda} \epsilon_{n}$. In addition, if the temperature transforms as $T \rightarrow e^{-2 \lambda} T$, then the occupation probability $a_{n}=Z^{-1} e^{-\epsilon_{n} / T}$, and $Z=\sum_{i} e^{-\epsilon_{i} / T}$ remains invariant under such scaling transformations, and so does the entropy $S=-\sum a_{i} \ln a_{i}$, i.e.,

$$
\begin{aligned}
0=d S= & \left.\frac{\partial S}{\partial V}\right|_{T, H, c} d V+\left.\frac{\partial S}{\partial T}\right|_{V, H, c} d T \\
& +\left.\frac{\partial S}{\partial H}\right|_{V, T, c} d H+\left.\frac{\partial S}{\partial c}\right|_{V, T, H} d c .
\end{aligned}
$$

We note that after the above transformations, the system remains in thermal equilibrium. Substituting the scaling transformations into the above equation and noticing $V=L^{d}$, with $d$ being the dimension of the system, we obtain an important identity,

$\left.d V \frac{\partial S}{\partial V}\right|_{T, H, c}=\left.2 T \frac{\partial S}{\partial T}\right|_{V, H, c}+\left.2 H \frac{\partial S}{\partial H}\right|_{V, T, c}-\left.\chi c \frac{\partial S}{\partial c}\right|_{V, T, H}$,

that relates the entropy changes due to the variations of the interaction, magnetic field, and the volume of the system.
Using the definitions of GPs given in Eqs. (3), (8), (11), we obtain a simple identity:

$$
d \Gamma+2 \Gamma_{\text {mag }}-\chi \Gamma_{\text {int }}=2 .
$$

In one-dimensional systems we have $d=1$ and $\chi=-1$ [39]; the identity above is reduced to $\Gamma+2 \Gamma_{\text {mag }}+\Gamma_{\text {int }}=2$. This relation is valid in any dimension and can also be proven using standard statistical mechanical considerations (see [19]). For a three-dimensional free gas [40], it is clear that $\Gamma_{\mathrm{mag}}=\Gamma_{\mathrm{int}}=$ 0 and thus $\Gamma=2 / d$. A further study of the identity (13) will be published elsewhere [41].

\section{APPLICATIONS: QUANTUM CRITICALITY AND QUANTUM REFRIGERATION}

Inspecting Eq. (10), it is possible to lower the temperature of the system by changing the external magnetic field in an isentropic process. The efficiency of cooling is also related to the magnetic Grüneisen parameter. In fact, it has been possible to cool the systems into extremely low temperatures via either spin flip or magnetic field gradient (spin transport) [36,37]. Other refrigerators have also been discussed $[42,43]$; see also discussions on cooling and thermometry of atomic Fermi gases [44].

In cold atomic gas systems, however, interconversion between different spin (hyperfine) states is very slow, and the usual magnetic cooling is inefficient. In addition, the corresponding external magnetic field is determined by population imbalance of the two spin states and cannot be controlled directly in experiments. On the other hand, by analogy between (10) and (12), it should be possible to lower the temperature of the system by changing the interaction strength in an isentropic process. This offers particular convenience since in most cold-atom systems, interactions can be controlled via Feshbach resonances.

\section{A. Interaction-driven refrigeration}

To make the above statement concrete, we first demonstrate quantum refrigeration based on the Bethe ansatz solution of the Lieb-Liniger model, which describes the 1D Bose gas with a contact interaction. The Hamiltonian of the Lieb-Liniger model in a $1 \mathrm{D}$ box with length $L$ is given by [45]

$$
\hat{H}=-\frac{\hbar^{2}}{2 m} \sum_{i=1}^{N} \frac{\partial^{2}}{\partial x_{i}^{2}}+2 c \sum_{1 \leqslant i<j \leqslant N} \delta\left(x_{i}-x_{j}\right),
$$

where $m$ is the mass of the particles and $c$ is the coupling strength, which is determined by the 1D scattering length $c=$ $-2 \hbar^{2} / m a_{1 D}$. In a quasi-1D system, $a_{1 D}=\left(-a_{\perp}^{2} / 2 a_{s}\right)[1-$ $\left.C\left(a_{s} / a_{\perp}\right)\right][39,46,47]$, where $a_{\perp}$ is the oscillator length in the transverse direction, $a_{s}$ is the three-dimensional scattering length, and $C$ is a constant.

Before analyzing the refrigerator cycle, we first briefly review the scaling invariance of this model. The Hamiltonian (14) can be solved by Bethe ansatz [45,48], and here we list some related key results in the Supplemental Material [19]. Suppose that we have obtained the solution of the thermodynamic Bethe ansatz equation of dressed energy $\epsilon(k)$ under the input parameters $\mu, T$, and $c$ [49]; 

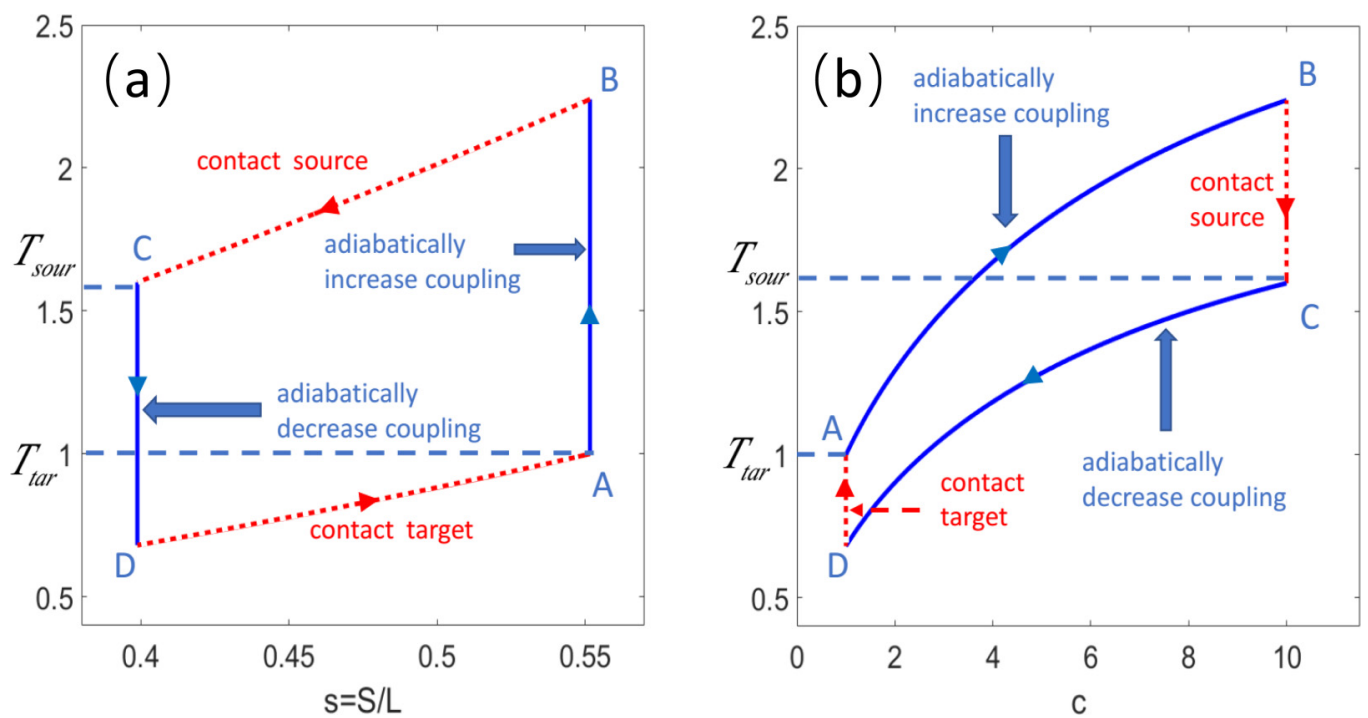

FIG. 1. Interaction-driven refrigeration cycle with the Lieb-Linger model in (a) the $T-s$ plane and (b) $T$-c plane. The cycle consisting of four processes is an analog of the demagnetization refrigeration which we discussed in [19]. Here the processes are $A \rightarrow B$, interaction ramp-up isentrope; $B \rightarrow C$, an isochore by contacting a hot source (release heat to the hot source); $C \rightarrow D$, interaction ramp-down isentrope; $D \rightarrow A$, an isochore by contacting a cold source (absorb heat from the target source). The cycle in both (a) and (b) is plotted by numerically solving the thermodynamic Bethe ansatz equations of the Lieb-Linger model (see [19]). The cycle begins at point $A$ with $n=0.1, c=1.0, T=1.0$ (here all the quantities are in the natural units $\hbar=2 m=k_{B}=1$ ). Then the coupling strength is tuned to the strong interacting region $c=10.0$; after contacting with the heat source the coupling strength is tuned back to $c=1.0$. Finally, the working material contacts the target sufficiently, and then a cycle is complete. This figure shows the complete analogy of the interaction-driven MCE to the traditional MCE.

it is obvious that the dressed energy $\epsilon^{\prime}\left(k^{\prime}\right)=e^{-2 \lambda} \epsilon\left(e^{\lambda} k^{\prime}\right)$ is the corresponding scaling form for input parameters under such rescaling $T^{\prime}=e^{-2 \lambda} T, \mu^{\prime}=e^{-2 \lambda} \mu$, and $c^{\prime}=e^{-\lambda} c$. Strictly speaking, the dressed energy is a homogeneous function with $\epsilon\left(e^{\lambda} k, e^{-2 \lambda} \mu, e^{-2 \lambda} T, e^{-\lambda} c\right)=e^{-2 \lambda} \epsilon(k, \mu, T, c)$ for all $\lambda \in R$. By definition, the pressure can be obtained in a straightforward way, $p\left(e^{-2 \lambda} \mu, e^{-2 \lambda} T, e^{-\lambda} c\right)=$ $e^{-3 \lambda} p(\mu, T, c)$ [19]. By differentiation, the density is given by $n\left(e^{-2 \lambda} \mu, e^{-2 \lambda} T, \mathrm{e}^{-\lambda} c\right)=e^{-\lambda} n(\mu, T, c)$. Furthermore, the entropy density $s=S / L$ is given by

$$
s\left(e^{-2 \lambda} \mu, e^{-2 \lambda} T, e^{-\lambda} c\right)=e^{-\lambda} s(\mu, T, c) .
$$

For the system with a fixed particle number, we need $L \rightarrow$ $L^{\prime}=e^{\lambda} L$ to ensure $N^{\prime}=N$ under scaling transformation $\mu^{\prime}=$ $e^{-2 \lambda} \mu, T^{\prime}=e^{-2 \lambda} T, c^{\prime}=e^{-\lambda} c$; then according to (15), we arrive at the conclusion that under this scaling transformation the entropy is unchanged, $S^{\prime}=S$, which is the key conclusion we used to obtain the identity of Grüneisen parameters (13) in the last section.

Similar discussions can also be had for the Gaudin-Yang model (see the next section) and other integrable models. However, we emphasize that the identity (13) depends only on the scaling properties of the spectrum. In fact, historically, the study of the Grüneisen parameter started from the discussion of homogeneity of thermodynamic quantities as functions of the oscillation frequency $\omega_{0}$ in the simple Einstein model $[1,2]$ (for details, see the Supplemental Material [19]).

Now let us return to our discuss of the refrigerator cycle driven by the interaction strength $c$ in the Lieb-Liniger model (14). As a direct analogy to the demagnetization cooling, the interaction-driven refrigerator cycle is shown in the
$T-S$ and $T-c$ planes in Fig. 1 via a rigorous calculation by the thermodynamic Bethe ansatz equations; details are given in the Supplemental Material [19]. In this approach, grand-canonical equilibrium states are valid for the study of thermodynamical properties of quantum gases trapped in a harmonic potential [50,51], although 1D tubes are well isolated from each other on a certain experimental timescale. We note that in the process of changing the interaction strength $c$, it is important at the same time to change the size of the system $\left(L \rightarrow L^{\prime}=e^{\lambda} L\right)$, so the spectrum of the system satisfies the scaling law, and thus, the process remains thermodynamically adiabatic under the quantum-mechanical unitary evolution.

Figure 1(a) shows the four strokes in a cooling cycle with the interacting bosons. For $A \rightarrow B$, the working medium is initially in the thermal state $A$ determined by the interaction strength $c_{A}=1$ and temperature $T_{\mathrm{tar}}=1$. The isentropic ramp up of interaction takes place, and the interaction strength is finally enhanced to the value $c_{B}$. After the adiabatic unitary evolution, the system reaches a state with temperature $T_{B}$. For $B \rightarrow C$, keeping $c_{B}$ constant, the working medium is coupled to the hot reservoir at temperature $T_{\text {sour }}$ and reaches the equilibrium state $\left(c_{B}, T_{\text {sour }}\right)$. The heat $\Delta Q_{1}$ is moved from the working medium to the hot reservoir. For $C \rightarrow D$, the working medium is decoupled from the hot reservoir. By performing external work, the interaction ramp-down isentropic process takes place. The interaction strength decreases from $c_{B}$ to $c_{D}=c_{A}$, and the working medium reaches the temperature $T_{D}$. For $D \rightarrow A$, the working medium is coupled to the target cold reservoir while keeping the interaction strength constant until it reaches the thermal state $\left(c_{A}, T_{\text {tar }}\right)$. The heat $\Delta Q_{2}$ is extracted from the target reservoir. The cooling efficiency is 
$\eta=\Delta Q_{2} / \Delta Q_{1}$. We would like to stress that in a realistic cycle, $A \rightarrow B$ and $C \rightarrow D$ are unlikely to be rigorously quantum adiabatic [52].

As discussed above, the temperatures at states $B$ and $D$ are still well defined if the spectra of the working system are scaling invariant under unitary evolution (see the discussion in Ref. [53]). The efficiency of such an interaction-driven cycle is set by the heat transfer between the target and reservoirs. An important practical concern of the cooling process is the maximal speed of interaction modulation below which the system can remain adiabatic, especially close to the quantum critical regime. In this regard, it has been proved by Campisi and Fazio [54] that the performance rate of the Otto engine can be enhanced when the working substance is at the verge of quantum criticality. This is mainly due to the emergence of new quantum scalings of the energy, entropy, and density which lead to the change in the speed of the propagation near a quantum critical point. This performance rate does not seem to directly relate to the known LiebRobinson bound in unitary evolution of quantum dynamics (see more discussions in [55-57]). In fact, the timescale in these discussions is under the typical timescale in quantum physics of the order $O(\hbar)$ [58], which can be ignored in quantum statistic physics because the Plank scale is much smaller than the Boltzmann scale. Therefore, it can be expected that the thermodynamic equilibrium takes place almost instantaneously.

The exact solution of the working system allows us to determine working efficiency in this particular case. We would like to mention that the modulation of the coupling strength in an interaction-driven cooling cycle can be associated with the coupling to external degrees of freedom (also see a recent study of the quantized refrigerator [59]). In low-temperature physics, the reachable low-temperature limit is the most important issue for engineering refrigeration. In the next section, we shall discuss the reachable lowest temperature for an engineering refrigeration with the $1 \mathrm{D}$ interacting fermions at quantum criticality.

\section{B. The Grüneisen parameter at quantum criticality}

As discussed in the last section, the Grüneisen parameters play a central role in this cooling process based on Eq. (10) or (12). Since the Grüneisen parameters are second-order derivatives with respect to free energy, it is expected that the Grüneisen parameters will also show divergent and scaling behaviors at the quantum critical points (QCPs) [13,14,17,18,28,33,35], leading to much enhanced effects for quantum refrigeration.

In order to illustrate this idea and to analyze the scaling behaviors of the GPs, we take the Yang-Gaudin model $[60,61]$ as an example to carry out rigorous calculations. This model was solved long ago by Yang [60] and Gaudin [61] using the Bethe ansatz. Theoretical prediction of the existence of a Fulde-Ferrell-Larkin-Ovchinnikov (FFLO) pairing state in the 1D interacting Fermi gas emerged by using the exact solution [62-64]. The key features of this $T=0$ phase diagram were experimentally confirmed using finite-temperature density profiles of trapped fermionic ${ }^{6} \mathrm{Li}$ atoms [50]. The

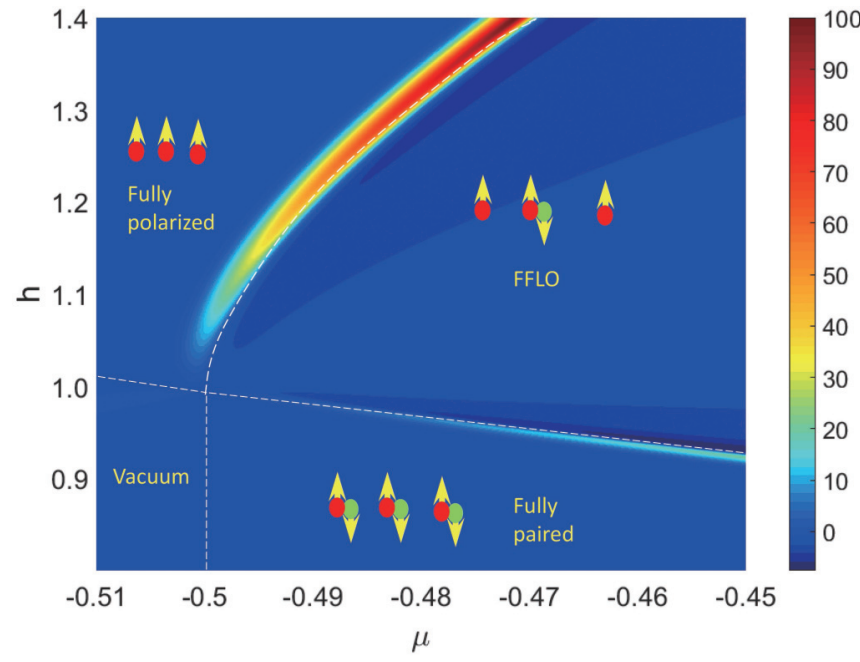

FIG. 2. Contour plot of the negative GP (3), i.e., $-\Gamma$, mapping out the full phase diagram of the Yang-Gaudin model with an attractive interaction in the $h-\mu$ plane. It consists of three novel phases, a fully paired state, a fully polarized state, and an FFLO-like state. Here the dimensionless temperature $t=0.001$. The GP has a sudden enhancement near the phase boundaries, giving a universal divergent scaling $\Gamma \sim t^{-1 / 2}$; in the numerical calculation of this figure, we assumed $c=1$, see the text.

Hamiltonian of the Yang-Gaudin model

$$
\begin{aligned}
\hat{H}= & \sum_{\sigma=\downarrow, \uparrow} \int \phi_{\sigma}^{\dagger}(x)\left(-\frac{\hbar^{2}}{2 m} \frac{d^{2}}{d x^{2}}+\mu_{\sigma}\right) \phi_{\sigma}(x) d x \\
& +g_{1 \mathrm{D}} \int \phi_{\downarrow}^{\dagger}(x) \phi_{\uparrow}^{\dagger}(x) \phi_{\uparrow}(x) \phi_{\downarrow}(x) d x \\
& -\frac{1}{2} h \int\left(\phi_{\uparrow}^{\dagger}(x) \phi_{\uparrow}(x)-\phi_{\downarrow}^{\dagger}(x) \phi_{\downarrow}(x)\right) d x
\end{aligned}
$$

describes a 1D $\delta$-function interacting two-component Fermi gas of $N$ fermions with mass $m$ and an external magnetic field $h$ constrained with periodic boundary conditions to a line of length $L . g_{1 D}=-2 \hbar^{2} /\left(m a_{1 D}\right)$ is determined by an effective scattering length $a_{1 D}$ via Feshbach resonances or confinement-induced resonances [39,46,47]. $g_{1 D}>0(<0)$ represents repulsive (attractive) interaction. Usually, $c=$ $m g_{1 D} / \hbar^{2}=-2 / a_{1 D}$ denotes the effective interaction strength.

Here we show that the different GPs, (3), (9), and (11), not only signal quantum phase transitions but also quantify various fluctuations in quantum systems. Using the exact thermodynamic Bethe ansatz (TBA) equations, a full critical phase diagram of the Yang-Gaudin model at $t=0.0001 \epsilon_{b}$ is determined by the GP expression (3) (see Supplemental Material [19]). In this contour plot, the rescaled units were used, i.e., $\tilde{t}=t /\left(c^{2} / 2\right), \tilde{\mu}=\mu /\left(c^{2} / 2\right)$, and $\tilde{h}=h /\left(c^{2} / 2\right)$. We observe that the GP (3) characterizes the universal divergent scaling near the phase boundaries. It shows that the energypressure covariance has stronger fluctuations than the energy fluctuation. This feature can be used to identify different quantum phases, i.e., novel Luttinger liquids of the fully paired state, a FFLO-like pairing state, and a fully polarized state (see Fig. 2). We show that the phase boundaries between the fully polarized phase and FFLO-like pairing phase and between the 
fully paired phase and FFLO-like pairing phase in Fig. 2 can be cast into a universal scaling form (for a constant $h$ ):

$$
\Gamma=\sqrt{\lambda \pi} n t^{-1 / 2} \mathcal{G}\left(\frac{\lambda\left(\mu-\mu_{c}\right)}{t}\right),
$$

with the factor $\lambda=1$ and $\lambda=2$ for phase transitions from FFLO-like to fully pairing and fully polarized to FFLO-like, respectively. In the above equation, $n$ is the density, $\mathcal{G}(x)$ is the scaling function, and $\mu$ is an effective chemical potential [24]. A more detailed study on the quantum scalings of the GPs (17) was published elsewhere [41]. In addition, the use of magnetic and interacting GPs (9) and (11) also gives the same phase diagram at low temperatures.

The divergence of the GPs at $T \rightarrow 0$ near QCPs can be clearly understood by investigating the entropy of the system. At low temperatures, the state of the system away from the critical points usually behaves like a Fermi liquid (or a Tomonaga-Luttinger liquid region in 1D; see Fig. 2). The entropy $S \propto T$. In contrast, the entropy at the quantum criticality behaves as $[65,66]$

$$
\frac{S}{V} \propto T^{(d / z)+1-(1 / v z)} \mathcal{K}\left(\frac{\mu-\mu_{c}}{T^{1 / v z}}\right) .
$$

For a 1D system, the dynamic critical exponent $z=2$, and the critical exponent for correlation length $v=1 / 2$, whereas $\mu$ presents an effective chemical potential and $\mu_{c}$ is the quantum critical point. $\mathcal{K}(x)$ is some analytical scaling function. Note that the entropy is exactly zero at zero temperature, so there is no constant term in Eq. (18) [21,22,24,38,67,68]. For the Gaudin-Yang model, the entropy $S \propto \sqrt{T} \gg T$ near the QCPs, which implies the local maximum of the entropy at QCPs. If we plot the entropy in the $T-H$ plane in Fig. 3, the isentropic lines will be bent down significantly at QCPs. A similar feature is observed in the $T-|c|$ plane (see Fig. S2 in [19]). According to Eqs. (10) and (12), the GPs are proportional to the slope of the isentropic line, which leads to the divergence of the GP when $T \rightarrow 0$.

We note that the maximum point of the entropy is shifted from the critical point when temperature increases. The local maximum of the entropy at low temperatures reveals the essence of the QCPs when the low-lying excitation becomes degenerate with the ground state [69]. In general, the divergence of the GPs is also present in generic models when a quantum phase transition occurs and has been extensively studied both in theory and experiments [10-16,18,25,27,33$35,70,71]$

\section{Refrigeration near a quantum phase transition}

For refrigeration, it is important to ask what the lowest temperature one can achieve is. In the Supplemental Material [19], we answer this question for the free Fermi gas. This question seems trivial in common refrigeration [27]. However, if the system approaches its quantum critical point, things can be significantly different. The divergent behavior of the GPs near QCPs can lead to significant cooling of the system. In fact, the feature of local maximum of the entropy leads to a local temperature minimum in an isentropic process (see Fig. 3). Consequently, one can make use of this fact to enhance the MCE (or interaction-driven cooling). Using the exact solution of the 1D attractive Fermi gas, we further

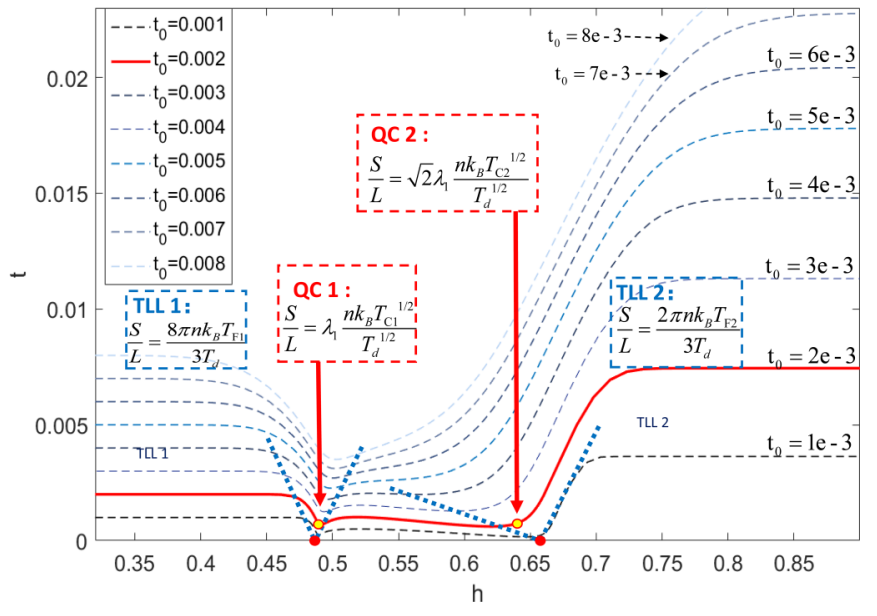

FIG. 3. The contour plot of the entropy in the $t-h$ plane for the attractive Yang-Gaudin model at low temperatures. Here the magnetic field $h=H / \epsilon_{b}$ and the temperature $t=T / \epsilon_{b}$ are rescaled by the binding energy with $2 m=\hbar=k_{B}=1$. We carried out our calculation through the TBA equations [19] with a fixed density $n=0.1$. $h_{c 1}$ and $h_{c 2}$ are the critical points for the phase transitions from fully paired TLL to the FLLO-like phase and from the FFLO-like phase to the fully polarized phase at $t=0$, respectively. The dashed lines in different colors present the contour values of entropies at different temperatures. The bending down of the contour lines indicates an entropy accumulation with a minimum temperature (yellow dot). For $h<h_{c 1}$ the system is in the TLL of bound pairs obeying the state equation (22), whereas for $h>h_{c 2}$ the system is in a fully polarized TLL obeying Eq. (22). These analytical results of the state equations directly give the minima of the temperature during the adiabatic demagnetization processes [see (23)].

demonstrate magnetic (or interaction-driven) refrigeration in the interacting Fermi gas [60,61].

Let us take an example of magnetic-driven cooling. Using the condition [see Eq. (10)]

$$
\Gamma_{\text {mag }}=0 \text {, }
$$

we may answer what the lowest possible temperature achievable is. For the Yang-Gaudin model [19], we expect an enhancement of the cooling efficiency when the working system is approaching a quantum critical point in the phase diagram (Fig. 2). Here we focus on the low-temperature region, i.e., $T \ll T_{d}$, where $T_{d}=\left(\frac{\hbar^{2} n^{2}}{2 m k_{B}}\right)$ is the degenerate temperature. Figure 3 shows that the condition $\Gamma_{\text {mag }}=0$ gives solutions for each quantum phase transition. Like the free Fermi gas given in [19], the condition $\Gamma_{\mathrm{mag}}=0$ leads to two independent equations for the Yang-Gaudin model at the two quantum critical points, namely,

$$
-\frac{1}{2} \operatorname{Li}_{\frac{1}{2}}\left(-e^{\tilde{A}^{(r)} / t}\right)+\frac{\tilde{A}^{(r)}}{t} \mathrm{Li}_{-\frac{1}{2}}\left(-e^{\tilde{A}^{(r)} / t}\right)=0,
$$

where $r=1$ and $r=2$ stand for the unpaired fermions and bound pairs, respectively. This means that at the phase transition $H_{c 1}$ the density of states of unpaired fermions changed dramatically, whereas at the critical point $H_{c 2}$ the density of states of the paired fermions changed dramatically. The effective chemical potentials of unpaired fermions and pairs, $\tilde{A}^{(1)}=$ $(\mu+H / 2) / \epsilon_{b}$ and $\tilde{A}^{(2)}=\left(2 \mu+c^{2} / 2\right) / \epsilon_{b}$, were rescaled by 
the bonding energy $\epsilon_{b}=c^{2} / 2$. Here $\mu$ is the chemical potential, and $t=T / \epsilon_{b}$ is the rescaled temperature.

Equation (20) is very similar to the equation $\mathcal{Y}(x)=x-$ $\frac{\mathrm{Li}_{1 / 2}\left(-e^{x}\right)}{2 \mathrm{Li}_{-1 / 2}\left(-e^{x}\right)}=0$ found for the free Fermi gas [19]. We thus have the same solution, $\tilde{A}^{(r)} / t=x_{0} \approx 1.3117$. Substituting this solution into TBA results given in [19], we get entropies at the phase transition points from the fully paired phase to the FFLO-like state and from the FFLO-like state to the fully paired Fermi gas, respectively,

$$
\begin{aligned}
& \frac{S}{L}=\lambda_{1} \frac{\sqrt{m}}{\hbar \sqrt{2 \pi}} k_{B}^{3 / 2} T_{c 1}^{1 / 2}, \quad H \rightarrow H_{m 1}, \\
& \frac{S}{L}=\lambda_{1} \frac{\sqrt{m}}{\hbar \sqrt{\pi}} k_{B}^{3 / 2} T_{c 2}^{1 / 2}, \quad H \rightarrow H_{m 2},
\end{aligned}
$$

where $\lambda_{1}=x_{0} \operatorname{Li}_{1 / 2}\left(-e^{x_{0}}\right)-\frac{3}{2} \operatorname{Li}_{3 / 2}\left(-e^{x_{0}}\right) \approx 1.3467 . H_{m 1}$ and $H_{m 2}$ are two critical fields corresponding to the two temperature minima in the isentropic contour lines. Using the TBA equation, we have the entropy in the liquid phases of pairs and fully polarized fermions,

$$
\begin{array}{ll}
\frac{S}{L}=\frac{4 m}{3 \hbar^{2}} k_{B}^{2} T_{L 1} n^{-1}, & H<H_{m 1}, \\
\frac{S}{L}=\frac{m}{3 \hbar^{2}} k_{B}^{2} T_{L 2} n^{-1}, & H>H_{m 2} .
\end{array}
$$

Here $T_{L 1}$ and $T_{L 2}$ are the temperatures in the Luttinger liquid regions; see phases TLL 1 and TLL 2 in Fig. 3. For the first equation in (22), we applied the strong-coupling condition $\gamma=c / n \gg 1$. From Eqs. (21) and (22), we find two temperature minima of the refrigeration around the two phase transitions:

$$
\begin{aligned}
& \frac{T_{c 1}}{T_{d}}=8 \lambda_{2}^{2}\left(\frac{T_{L 1}}{T_{d}}\right)^{2}, \\
& \frac{T_{c 2}}{T_{d}}=\frac{\lambda_{2}^{2}}{2}\left(\frac{T_{L 2}}{T_{d}}\right)^{2},
\end{aligned}
$$

with $\lambda_{2}=\frac{2 \pi}{3 \lambda_{1}} \approx 1.5552$. We further observe that the leading contribution to the entropy at the critical point $H_{m 1}$ involves the excitations of the excess fermions [21]. However, at the critical point $H_{m 2}$, the leading contribution to the entropy comes from the excitations of the bound pairs. In the isentropic process, the system can thus retain more entropy per unit temperature near the finite-temperature critical point $H_{m 2}$. This result reveals an enhancement of the cooling efficiency at $H_{m 2}$. In cold-atom experiments, the temperature is usually much lower than the degenerate temperature [72], i.e., $T_{L 1} / T_{d} \ll 1$ and $T_{L 2} / T_{d} \ll 1$. From Eq. (23), we thus have $T_{c 1} \ll T_{L 1}$ and $T_{c 2} \ll T_{L 2}$. Moreover, the ideal limit of the temperature $T_{c_{1,2}}$ for the refrigeration is one order of magnitude lower than the temperature at the heat source $T_{L_{1,2}}$.

\section{SUMMARY}

We have conducted a comprehensive investigation of the Grüneisen parameters for ultracold quantum gases, including its origin, an identity, the caloric effects, and quantum refrigeration. We have proposed the interaction-related GP, which reveals also the characteristic energy scales of a quantum system induced by the variation of the interaction. Together with the other two GPs related to the variations of volume and magnetic field, we have established an identity among them which characterizes the universal scalings of fluctuations and the caloric effect in quantum gases. Based on the entropy accumulation at the quantum critical point, two promising protocols of quantum refrigeration driven either by interaction or by magnetic field were studied. Using the Bethe ansatz, we studied the expansionary, magnetic, and interacting GPs; quantum refrigeration; the magnetocaloric effect; and the quantum critical phenomenon of the Lieb-Liniger model and Yang-Gaudin model. Our method opens an avenue to further study the GPs and quantum refrigeration for quantum gases with different spin symmetries in 1D and higher dimensions.

\section{ACKNOWLEDGMENTS}

The author thanks Y. X. Liu, L. Peng, Y. Z. Jiang, Y. Y. Chen, F. He, H. Pu, and R. Hulet for helpful discussions. This work is supported by National Key R\&D Program of China Grant No. 2017YFA0304500, the key NSFC Grant No.11534014, and NSFC Grants No. 11804377 and No. 11874393 . S.Z. is supported by HK GRF 17318316 , 17305218 and CRF C6026-16W and C6005-17G and the Croucher Foundation under the Croucher Innovation Award. Y.-C.Y. thanks the University of Hong Kong for kind hospitality. X.-W.G. acknowledges Rice University for supporting his visit.
[1] E. Grüneisen, Über die thermische Ausdehnung und die spezifische Wärme der Metalle, Ann. Phys. (Berlin, Ger.) 331, 211 (1908).

[2] E. Grüneisen, Theorie des festen Zustandes einatomiger elemente, Ann. Phys. (Berlin, Ger.) 344, 257 (1912).

[3] F. D. Stacey, Theory of thermal and elastic properties of the lower mantle and core, Phys. Earth Planet. Inter. 89, 219 (1995).

[4] J. Shanker, K. Sunil, and B. S. Sharma, The Grüneisen parameter and its higher order derivatives for the earth lower mantle and core, Phys. Earth Planet. Inter. 262, 41 (2017).

[5] P. Mausbach, A. Koster, G. Rutkai, M. Thol, and J. Vrabec, Comparative study of the Grüneisen parameter for 28 pure fluids, J. Chem. Phys. 144, 244505 (2016).
[6] G. Liu, J. Zhou, and H. Wang, Anisotropic thermal expansion of SnSe from first-principles calculations based on Grüneisen's theory, Phys. Chem. Chem. Phys. 19, 15187 (2017).

[7] L. Wang, M. T. Dove, K. Trachenko, Y. D. Fomin, and V. V. Brazhkin, Supercritical Grüneisen parameter and its universality at the Frenkel line, Phys. Rev. E 96, 012107 (2017).

[8] S. Kumar, S. K. Sharma, and O. P. Pandey, Brief report: Volume dependence of Grüneisen parameter for solids under extreme compression, Pramana 87, 21 (2016).

[9] S. A. Khrapak, Grüneisen parameter for strongly coupled Yukawa systems, Phys. Plasmas 24, 043706 (2017).

[10] R. Kuchler, N. Oeschler, P. Gegenwart, T. Cichorek, K. Neumaier, O. Tegus, C. Geibel, J. A. Mydosh, F. Steglich, L. 
Zhu, and Q. Si, Divergence of the Grüneisen Ratio at Quantum Critical Points in Heavy Fermion Metals, Phys. Rev. Lett. 91, 066405 (2003).

[11] R. Küchler, P. Gegenwart, J. Custers, O. Stockert, N. CarocaCanales, C. Geibel, J. G. Sereni, and F. Steglich, Quantum Criticality in the Cubic Heavy-Fermion System CeIn ${ }_{3-x} \mathrm{Sn}_{x}$, Phys. Rev. Lett. 96, 256403 (2006).

[12] R. Küchler, P. Gegenwart, C. Geibel, and F. Steglich, Systematic study of the Grüneisen ratio near quantum critical points, Sci. Technol. Adv. Mater. 8, 428 (2007).

[13] P. Gegenwart, Grüneisen parameter studies on heavy fermion quantum criticality, Rep. Prog. Phys. 79, 114502 (2016).

[14] A. Steppke, R. Küchler, S. Lausberg, E. Lengyel, L. Steinke, R. Borth, T. Lühmann, C. Krellner, M. Nicklas, C. Geibel, F. Steglich, and M. Brando, Ferromagnetic quantum critical point in the heavy-fermion metal $\mathrm{YbNi}_{4}\left(\mathrm{P}_{1-x} \mathrm{As}_{x}\right)$, Science 339, 933 (2013).

[15] Y. Tokiwa, T. Radu, C. Geibel, F. Steglich, and P. Gegenwart, Divergence of the Magnetic Grüneisen Ratio at the FieldInduced Quantum Critical Point in $\mathrm{YbRh}_{2} \mathrm{Si}_{2}$, Phys. Rev. Lett. 102, 066401 (2009).

[16] B. Wolf, Y. Tsui, D. Jaiswal-Nagar, U. Tutsch, A. Honecker, K. Removiç-Langer, G. Hofmann, A. Prokofiev, W. Assmus, G. Donath, and M. Lang, Magnetocaloric effect and magnetic cooling near a field-induced quantum-critical point, Proc. Natl. Acad. Sci. USA 108, 6862 (2011).

[17] B. Wolf, A. Honecker, W. Hofstetter, U. Tutsch, and M. Lang, Cooling through quantum criticality and many-body effects in condensed matter and cold gases, Int. J. Mod. Phys. B 28 1430017 (2014).

[18] H. Ryll, K. Kiefer, C. Ruegg, S. Ward, K. W. Kramer, D. Biner, P. Bouillot, E. Coira, T. Giamarchi, and C. Kollath, Magnetic entropy landscape and Grüneisen parameter of a quantum spin ladder, Phys. Rev. B 89, 144416 (2014).

[19] See Supplemental Material at http://link.aps.org/supplemental/ 10.1103/PhysRevResearch.2.043066 for a detailed study of the Grüneisen parameter, the identity, quantum refrigeration, and an introduction to the thermodynamic Bethe ansatz equations for the 1D Bose gas and spin-1/2 Fermi gas.

[20] K. G. Wilson, The renormalization group: Critical phenomena and the Kondo problem, Rev. Mod. Phys. 47, 773 (1975).

[21] Y.-C. Yu, Y.-Y. Chen, H.-Q. Lin, R. A. Römer, and X.-W. Guan, Dimensionless ratios: Characteristics of quantum liquids and their phase transitions, Phys. Rev. B 94, 195129 (2016).

[22] F. He, Y.-Z. Jiang, Y.-C. Yu, H.-Q. Lin, and X.-W. Guan, Quantum criticality of spinons, Phys. Rev. B 96, 220401(R) (2017).

[23] C. Chin, R. Grimm, P. Julienne, and E. Tiesinga, Feshbach resonances in ultracold gases, Rev. Mod. Phys. 82, 1225 (2010).

[24] X.-W. Guan, M. T. Batchelor, and C.-H. Lee, Fermi gases in one dimension: From Bethe ansatz to experiments, Rev. Mod. Phys. 85, 1633 (2013).

[25] L. J. Zhu, M. Garst, A. Rosch, and Q. M. Si, Universally Diverging Grüneisen Parameter and the Magnetocaloric Effect Close to Quantum Critical Points, Phys. Rev. Lett. 91, 066404 (2003).

[26] Y. Takahashi and H. Nakano, Magnetovolume effect of itinerant electron ferromagnets, J. Phys.: Condens. Matter 18, 521 (2006).

[27] A. Smith, C. R. H. Bahl, R. Bjørk, K. Engelbrecht, K. K. Nielsen, and N. Pryds, Materials challenges for high per- formance magnetocaloric refrigeration devices, Adv. Energy Mater. 2, 1288 (2012).

[28] S. B. Mousumi and P. Pankaj, Study of magnetic entropy and heat capacity in ferrimagnetic $\mathrm{Fe}_{3} \mathrm{Se}_{4}$ nanorods, J. Phys. D 49, 195003 (2016).

[29] O. Breunig, M. Garst, A. Klümper, J. Rohrkamp, M. M. Turnbull, and T. Lorenz, Quantum criticality in the spin-1/2 Heisenberg chain system copper pyrazine dinitrate, Sci. Adv. 3, eaao3773 (2017).

[30] Z. J. Liu, T. Song, X. W. Sun, Q. Ma, T. Wang, and Y. Guo, Thermal expansion, heat capacity and Grüneisen parameter of iridium phosphide $\mathrm{Ir}_{2} \mathrm{P}$ from quasi-harmonic Debye model, Solid State Commun. 253, 19 (2017).

[31] Here we do not write out explicitly the vertical lines and corresponding variables in the partial derivatives, where the derivatives are calculated in the grand-canonical ensemble with variables $V$ (volume), $T$ (temperature), $H$ (magnetic field), and $c$ (coupling strength).

[32] L. D. Landau and E. M. Lifshitz, Statistical Physics, Course of Theoretical Physics (Elsevier, 1986), Vol. 5.

[33] F. Weickert, R. Küchler, A. Steppke, L. Pedrero, M. Nicklas, M. Brando, F. Steglich, M. Jaime, V. S. Zapf, A. Paduan-Filho, K. A. Al-Hassanieh, C. D. Batista, and P. Sengupta, Lowtemperature thermodynamic properties near the field-induced quantum critical point in $\mathrm{NiCl}_{2}-4 \mathrm{SC}\left(\mathrm{NH}_{2}\right)_{2}$, Phys. Rev. B 85, 184408 (2012).

[34] M. Garst and A. Rosch, Sign change of the Grüneisen parameter and magnetocaloric effect near quantum critical points, Phys. Rev. B 72, 205129 (2005).

[35] D. Straßel, P. Kopietz, and S. Eggert, Magnetocaloric effects, quantum critical points, and the Berezinsky-Kosterlitz-Thouless transition in two-dimensional coupled spin-dimer systems, Phys. Rev. B 91, 134406 (2015).

[36] P. Medley, D. M. Weld, H. Miyake, D. E. Pritchard, and W. Ketterle, Spin Gradient Demagnetization Cooling of Ultracold Atoms, Phys. Rev. Lett. 106, 195301 (2011).

[37] D. M. Weld, H. Miyake, P. Medley, D. E. Pritchard, and W. Ketterle, Thermometry and refrigeration in a two-component Mott insulator of ultracold atoms, Phys. Rev. A 82, 051603 (2010).

[38] Y.-Y. Chen, Y.-Z. Jiang, X.-W. Guan, and Q. Zhou, Critical behaviors of contact near phase transitions, Nat. Commun. 5, 5140 (2014).

[39] M. Olshanii, Atomic Scattering in the Presence of an External Confinement and a Gas of Impenetrable Bosons, Phys. Rev. Lett. 81, 938 (1998).

[40] M. de Souza, P. Menegasso, R. Paupitz, A. Seridonio, and R. E. Lagos, Grüneisen parameter for gases and superfluid helium, Eur. J. Phys. 37, 055105 (2016).

[41] L. Peng, Y. Yu, and X.-W. Guan, Grüneisen parameters for the Lieb-Liniger and Yang-Gaudin models, Phys. Rev. B 100, 245435 (2019).

[42] B. Ekkes, Developments in magnetocaloric refrigeration, J. Phys. D 38, R381 (2005).

[43] V. V. Khovaylo, V. V. Rodionova, S. N. Shevyrtalov, and V. Novosad, Magnetocaloric effect in "reduced" dimensions: Thin films, ribbons, and microwires of Heusler alloys and related compounds, Phys. Status Solidi B 251, 2104 (2014).

[44] R. Onofrio, Cooling and thermometry of atomic Fermi gases, Phys. Our Days 59, 1129 (2016). 
[45] E. H. Lieb and W. Liniger, Exact analysis of an interacting Bose gas. I. The general solution and the ground state, Phys. Rev. 130, 1605 (1963).

[46] V. Dunjko, V. Lorent, and M. Olshanii, Bosons in Cigar-Shaped Traps: Thomas-Fermi Regime, Tonks-Girardeau Regime, and in Between, Phys. Rev. Lett. 86, 5413 (2001).

[47] M. Olshanii and V. Dunjko, Short-Distance Correlation Properties of the Lieb-Liniger System and Momentum Distributions of Trapped One-Dimensional Atomic Gases, Phys. Rev. Lett. 91, 090401 (2003).

[48] E. H. Lieb and F. Y. Wu, Absence of Mott Transition in an Exact Solution of the Short-Range, One-Band Model in One Dimension, Phys. Rev. Lett. 20, 1445 (1968).

[49] C. N. Yang and C. P. Yang, Thermodynamics of a onedimensional system of bosons with repulsive delta-function interaction, J. Math. Phys. 10, 1115 (1969).

[50] Y. Liao, A. S. C. Rittner, T. Paprotta, W. Li, G. B. Partridge, R. G. Hulet, S. K. Baur, and E. J. Mueller, Spin-imbalance in a one-dimensional Fermi gas, Nature (London) 467, 567 (2010).

[51] B. Yang, Y.-Y. Chen, Y.-G. Zheng, H. Sun, H.-N. Dai, X.-W. Guan, Z.-S. Yuan, and J.-W. Pan, Quantum Criticality and the Tomonaga-Luttinger Liquid in One-Dimensional Bose Gases, Phys. Rev. Lett. 119, 165701 (2017).

[52] In this refrigeration cycle, the adiabatic process refers to the process with $d S=0$ rather than the quantum adiabatic, where the time evolution of the system is under a unitary transformation introduced by the time-evolution operators. See the discussion in [53].

[53] Y.-Y. Chen, G. Watanabe, Y.-C. Yu, X.-W. Guan, and A. del Campo, An interaction-driven many-particle quantum heat engine and its universal behaviour, npj Quantum Inf., 5, 88 (2019).

[54] M. Campisi and R. Fazio, The power of a critical heat engine, Nat. Commun. 7, 11895 (2016).

[55] D. Poulin, Lieb-Robinson Bound and Locality for General Markovian Quantum Dynamics, Phys. Rev. Lett. 104, 190401 (2010).

[56] B. Nachtergaele, A. Vershynina, and V. A. Zagrebnov, LiebRobinson bounds and existence of the thermodynamic limit for a class of irreversible quantum dynamics, AMS Contemp. Math. 552, 161 (2011).

[57] B. Descamps, Asymptotically decreasing Lieb-Robinson velocity for a class of dissipative quantum dynamics, J. Math. Phys. 54, 092202 (2013).
[58] J.-S. Bernier, R. Tan, L. Bonnes, C. Guo, D. Poletti, and C. Kollath, Light-Cone and Diffusive Propagation of Correlations in a Many-Body Dissipative System, Phys. Rev. Lett. 120, 020401 (2018).

[59] W. Niedenzu, I. Mazets, G. Kurizki, and F. Jendrzejewski, Quantized refrigerator for an atomic cloud, Quantum 3, 155 (2019).

[60] C. N. Yang, Some Exact Results for the Many-Body Problem in One Dimension with Repulsive Delta-Function Interaction, Phys. Rev. Lett. 19, 1312 (1967).

[61] M. Gaudin, Un systeme a une dimension de fermions en interaction, Phys. Lett. A 24, 55 (1967).

[62] G. Orso, Attractive Fermi Gases with Unequal Spin Populations in Highly Elongated Traps, Phys. Rev. Lett. 98, 070402 (2007).

[63] H. Hu, X.-J. Liu, and P. D. Drummond, Phase Diagram of a Strongly Interacting Polarized Fermi Gas in One Dimension, Phys. Rev. Lett. 98, 070403 (2007).

[64] M. T. Guan, X. W. Batchelor, C. Lee, and M. Bortz, Phase transitions and pairing signature in strongly attractive Fermi atomic gases, Phys. Rev. B 76, 085120 (2007).

[65] X.-W. Guan and T.-L. Ho, Quantum criticality of a onedimensional attractive Fermi gas, Phys. Rev. A 84, 023616 (2011).

[66] M. P. A. Fisher, P. B. Weichman, G. Grinstein, and D. S. Fisher, Boson localization and the superfluid-insulator transition, Phys. Rev. B 40, 546 (1989).

[67] Y.-Z. Jiang, Y.-Y. Chen, and X.-W. Guan, Understanding manybody physics in one dimension from the Lieb-Liniger model, Chin. Phys. B 24, 050311 (2015).

[68] S. Cheng, Y.-C. Yu, M. T. Batchelor, and X.-W. Guan, FuldeFerrell-Larkin-Ovchinnikov correlation and free fluids in the one-dimensional attractive Hubbard model, Phys. Rev. B 97, 121111 (2018).

[69] S. Sachdev, Quantum Phase Transitions (Cambridge University Press, Cambridge, 2001).

[70] C. H. Lee and C. K. Gan, Anharmonic interatomic force constants and thermal conductivity from Grüneisen parameters: An application to graphene, Phys. Rev. B 96, 035105 (2017).

[71] S. Watanabe and K. Miyake, Grüneisen parameter and thermal expansion by the self-consistent renormalization theory of spin fluctuations, J. Phys. Soc. Jpn. 87, 034712 (2018).

[72] H. Pethick and C. J. Smith, Bose-Einstein Condensation in Dilute Gases, 2nd ed. (Cambridge University Press, Cambridge, 2008). 